\title{
eNeuro
}

Research Article: New Research / Sensory and Motor Systems

\section{Individualized Assays of Temporal Coding in the Ascending Human Auditory System}

https://doi.org/10.1523/ENEURO.0378-21.2022

Cite as: eNeuro 2022; 10.1523/ENEURO.0378-21.2022

Received: 16 September 2021

Revised: 12 January 2022

Accepted: 8 February 2022

This Early Release article has been peer-reviewed and accepted, but has not been through the composition and copyediting processes. The final version may differ slightly in style or formatting and will contain links to any extended data.

Alerts: Sign up at www.eneuro.org/alerts to receive customized email alerts when the fully formatted version of this article is published.

Copyright (C) 2022 Borjigin et al.

This is an open-access article distributed under the terms of the Creative Commons Attribution 4.0 International license, which permits unrestricted use, distribution and reproduction in any medium provided that the original work is properly attributed. 
$1 \quad$ Individualized Assays of Temporal Coding in the Ascending Human

2

3

4 ${ }^{5}{ }^{a}$ Weldon School of Biomedical Engineering, Purdue University, West Lafayette, IN, United States ${ }_{6}{ }^{\mathrm{b}}$ Department of Speech, Language, and Hearing Sciences, Purdue University, West Lafayette, IN, 7 United States

$8 \quad$ Number of pages: 30, Number of figures: 9, Number of tables: 1, Number of words for abstract: 233 ,

9 Number of words for introduction: 630, Number of words for discussion: 1092

10 Conflict of interests: There are no relevant financial or non-financial competing interests.

11 Acknowledgements: This work was supported by funding from the National Institutes of Health (Grant 12 No. R01DC015989).

\footnotetext{
${ }^{*}$ Correspondence: hbharadwaj@purdue.edu
} 


\begin{abstract}
Neural phase-locking to temporal fluctuations is a fundamental and unique mechanism by which acoustic information is encoded by the auditory system. The perceptual role of this metabolically expensive mechanism, the neural phase-locking to temporal fine structure (TFS) in particular, is debated. Although hypothesized, it is unclear if auditory perceptual deficits in certain clinical populations are attributable to deficits in TFS coding. Efforts to uncover the role of TFS have been impeded by the fact that there are no established assays for quantifying the fidelity of TFS coding at the individual level. While many candidates have been proposed, for an assay to be useful, it should not only intrinsically depend on TFS coding, but should also have the property that individual differences in the assay reflect TFS coding per se over and beyond other sources of variance. Here, we evaluate a range of behavioral and electroencephalogram (EEG)-based measures as candidate individualized measures of TFS sensitivity. Our comparisons of behavioral and EEG-based metrics suggest that extraneous variables dominate both behavioral scores and EEG amplitude metrics, rendering them ineffective. After adjusting behavioral scores using lapse rates, and extracting latency or percent-growth metrics from EEG, interaural timing sensitivity measures exhibit robust behavior-EEG correlations. Together with the fact that unambiguous theoretical links can be made relating binaural measures and phase-locking to TFS, our results suggest that these "adjusted" binaural assays may be well-suited for quantifying individual TFS processing. Key words: neural coding, temporal fine structure, electroencephalography, interaural time difference, frequency modulation, non-sensory factors
\end{abstract}

\title{
Significance Statement
}

The auditory system is unique among the senses in that neurons in the periphery fire precisely phaselocked spikes in response to fast temporal fluctuations in sound. Yet, the functional significance of this metabolically expensive initial neural code is debated. Here, we establish behavioral and physiological assays that can probe the fidelity of this phase-locking mechanism at the level of individual human subjects. These measures pave the way for future experiments that can more directly address foundational questions about the role of phase locking in everyday hearing, and test whether phase-locking deficits contribute to the listening difficulties in clinical populations. Importantly, our results also show that commonly used measures to assess phase locking are affected by extraneous variables, and thus ineffective. 


\section{Introduction}

44 All acoustic information we receive is conveyed through the firing rate and/or timing of the neural spikes

45 (i.e., rate-place vs temporal coding) of cochlear neurons. Temporal information in the basilar-membrane 46 vibrations consists of cycle-by-cycle variations in phase - the temporal fine structure (TFS), and dy47 namic variations in amplitude-the envelope (ENV) (Hilbert, 1906). Cochlear neurons phase-lock to both TFS (Johnson, 1980), and ENV (Joris and Yin, 1992) robustly, with TFS phase-locking extending 4 at least up to $1000 \mathrm{~Hz}$ (Verschooten et al., 2019). While the peripheral rate-place code has consistent counterparts throughout the auditory system, the upper limit of phase-locking progressively shifts to

lower frequencies along the ascending pathway (Joris et al., 2004). How this metabolically expensive initial/peripheral temporal code (Laughlin et al., 1998; Hasenstaub et al., 2010) contributes to everyday hearing, and how its degradation contributes to perceptual deficits, are foundational questions in auditory neuroscience and clinical audiology. Yet, the significance of TFS coding is debated (Oxenham, 2013; Drullman, 1995; Swaminathan and Heinz, 2012; Oxenham and Simonson, 2009).

Previous studies have explored whether sound localization and pitch perception benefit from TFS cues. While it is established that lateralization of low-frequency sounds depends on TFS (Smith et al., 2002; Yin and Chan, 1990), whether TFS is important for pitch perception is difficult to ascertain. Behavioral studies suggest that low-frequency periodic sounds elicit a stronger a pitch than high-frequency sounds (Moore, 1973; Houtsma and Smurzynski, 1990; Bernstein and Oxenham, 2003), suggesting a possible role for TFS. However, these results permit alternate interpretations in terms of place coding and harmonic resolvability (Oxenham, 2012). Regardless of its role in quiet, whether TFS is important for masking release in noise is further debated, especially when other redundant cues can also convey pitch or location, and when room reverberation can degrade temporal cues (Best et al., 2005; Oxenham and Simonson, 2009; Ihlefeld and Shinn-Cunningham, 2011).

To investigate the role of TFS, studies have used sub-band vocoding to independently manipulate ENV and TFS cues (Smith et al., 2002; Hopkins et al., 2008; Hopkins and Moore, 2009; Lorenzi et al., 2009; Ardoint and Lorenzi, 2010). However, acoustic manipulations cannot eliminate subsequent confounding of ENV, TFS, and place cues without detailed knowledge of cochlear processing at the individual level (Oxenham, 2013; Swaminathan and Heinz, 2012). Thus, establishing the precise role of TFS through vocoding experiments is difficult, although the use of high-fidelity vocoders can help (Viswanathan et al., 
2021). An alternative approach is to directly measure TFS sensitivity from individual listeners and compare it to individual differences in other perceptual measures. This approach has been successfully used to address other fundamental questions (McDermott et al., 2010; Bharadwaj et al., 2015; Whiteford et al., 2020). Unfortunately, the lack of established measures of TFS sensitivity at the individual level limits this enterprise.

Conventional behavioral TFS-sensitivity measurements have attempted to eliminate confounding cues such that primary task would rely on TFS processing (Strelcyk and Dau, 2009; Moore and Sek, 2009; Hopkins and Moore, 2010; Sek and Moore, 2012). However, they did not assess the influence of extraneous factors on the measured scores. Unfortunately, non-sensory factors can contribute significantly to individual variability even when the tasks themselves rely on specific acoustic cues (Kidd et al., 2007). Objective electrophysiological measures of TFS sensitivity can circumnavigate this problem; however, such studies are scarce (Verschooten et al., 2015; Parthasarathy et al., 2020). Here, we employ a battery of both behavioral and electroencephalography (EEG)-based measures of TFS sensitivity on a cohort of normal-hearing individuals to identify candidate assays of TFS processing at the individual level. Our results suggest that extraneous variables dominate both behavioral and raw EEG measures. However, with adjustments, we observed robust behavior-EEG correlations in binaural assays, rendering them well-suited for quantifying individual TFS processing.

\section{Materials and Methods}

The primary goal of the current study was to evaluate an array of both behavioral and electrophysiological measures as candidate assays of TFS sensitivity at the individual level. Based on the finding that nonsensory factors contribute significantly to behavioral TFS measures, a large-N supplementary behavioral experiment was conducted to assess whether non-sensory factors also influence ENV sensitivity when measured from naïve participants.

\subsection{Participants}

One hundred and fifty-three listeners, aged 18-60 years, were recruited from the local community near Purdue University. All human subject measures were conducted following protocols approved by the Purdue University Internal Review Board and the Human Research Protection Program. Participants 
were recruited via posted flyers and bulletin-board advertisements and provided informed consent. All participants had pure-tone air-conduction thresholds of $25 \mathrm{~dB}$ HL or better at octave frequencies from 500 to $8000 \mathrm{~Hz}$. Of the 153 subjects, 44 (20 males) participated in the main experiments designed to evaluate candidate assays of TFS processing. The remaining N=109 participated in the supplementary experiment aimed at testing whether non-sensory factors also influence ENV sensitivity. Although the goal of the main experiment was to conduct all behavioral and electrophysiological TFS measures on each participant, some were not able to finish the full study battery due to limited availability. Among the 44 listeners who participated in the main study, 43 completed the frequency modulation (FM) detection task, and 36 completed the interaural time difference (ITD) detection task. The intersection-33 subjects - completed both behavioral measurements. Among all participants $(\mathrm{n}=44), 42$ subjects completed EEG-ITD sensitivity measurements; 25 of those 42 subjects also completed EEG-FFR (frequency following response) measurements. Among the subjects who completed both behavioral measurements, all except one $(n=32)$ completed the EEG-ITD measurement; these subjects include all participants who completed the EEG-FFR measurements $(n=25)$. Therefore, the subjects who completed both behavioral measurements $(n=32$, age: mean $=26.8$, std $=11.2)$ were included for the main analyses including brainbehavior correlations. Although the wide age range, only 6 out of 33 subjects were older than 35 years at the time of the testing and age did not significantly correlate with any measure of this study.

\subsection{Experimental Design and Statistical Analyses}

\subsubsection{Behavioral Measures of the TFS Coding.}

Each of the following behavioral measurements was conducted on a different day from the others to randomize the influence of factors that may be idiosyncratic to a specific test day/session. A single lab visit contained only one behavioral measurement to reduce the impact of cognitive fatigue due to hour-long experiments.

Frequency Modulation (FM) Detection Thresholds. To obtain monaural TFS sensitivity, FM thresholds were measured separately in each ear, using a weighted (3:1) one-down-one-up (Kaernbach, 1991), two-alternatives-forced-choice (2AFC) adaptive procedure. The stimulus in the target interval was a $500-\mathrm{ms}-$ long $500-\mathrm{Hz}$ tone with frequency modulation at a $2-\mathrm{Hz}$ rate and variable depth. The reference interval was a $500 \mathrm{~Hz}$ pure tone. The inter-stimulus gap was $900 \mathrm{~ms}$. The stimulus was ramped on and 
off with a rise/fall time of $5 \mathrm{~ms}$ to exclude audible transitions. The stimulus level was $70 \mathrm{~dB}$ SPL. The subjects were instructed to press a button to indicate the interval containing the FM. Each measurement block was terminated after 11 reversals and the median of all the reversals from the adaptive procedure was extracted as the threshold. Four blocks of measurements were obtained in each ear from each subject. Except for an additional "demo" block to orient the participants before the formal testing, there was no further training. Sennheiser HDA 300 over-the-ear headphones were used for stimulus delivery. The slow FM rate of $2 \mathrm{~Hz}$ was chosen because it is thought that TFS cues are used to detect FM at rates below about $10 \mathrm{~Hz}$ (Moore and Sek, 1996; Strelcyk and Dau, 2009). However, recent evidence suggests that this may not be the case (Whiteford et al., 2020). Nonetheless, given the large body of literature using and interpreting slow-FM detection as a measure of TFS sensitivity, we chose to include this in the battery of candidate measures.

Interaural Time Difference (ITD) Detection Thresholds. To obtain a binaural measure of TFS sensitivity, we measured ITD detection thresholds using a three-down-one-up, two-alternatives-forcedchoice adaptive procedure. The stimulus consisted of two consecutive $400 \mathrm{~ms}-\mathrm{long}, 500 \mathrm{~Hz}$ tone bursts with an ITD. The leading ear for the ITD was switched from the first burst to the second. The stimulus was ramped on and off with a rise/fall time of $20 \mathrm{~ms}$ to exclude audible transitions and to reduce reliance on onset ITDs. The stimuli were presented at $70 \mathrm{~dB}$ SPL. Subjects were asked to report the direction of the jump (left-to-right or right-to-left) between the intervals through a button press. It was preferable to have subjects indicate the direction of change because absolute lateralization can be influenced by multiple factors (Moore and Sek, 2009). The threshold was defined as the geometric mean of the last nine reversals, and measured repeatedly across eight blocks, with a short break scheduled after the fourth block. Etymotic Research (ER-2) insert earphones were used for delivering the stimuli. A separate "demo" block was included before the experimental blocks to familiarize the subject with the task.

"Non-sensory" Score. Because the main goal of the study is to evaluate candidate measures of TFS coding in naïve subjects, i.e., individuals without extensive training/practice on the measured tasks, we anticipated that extraneous "non-sensory" variables may influence the measured thresholds. Accordingly, percent-incorrect scores on easy "catch" trials were calculated to quantify the subject's engagement. Errors made in these catch trials likely reflect non-sensory factors such as lapses in attention, variations in motivation, alertness, etc., rather than the strength of sensory coding. For the FM detection task, trials frequency deviations (modulation depths) greater than $15 \mathrm{~Hz}$ were deemed to be catch trials, and 
the percent-incorrect scores were calculated for just these trials for each subject as an estimate of lapse rate. Similarly, the criterion for designating a trial as a "catch" trial for the ITD detection task was that the ITD exceeded 80 microseconds. The number of catch trials available varied from subject to subject because of the adaptive nature of the task. On average, the FM and ITD detection tasks included 3-10 catch trials per block. To mitigate the influence of extraneous variables such as engagement and motivation on the measured thresholds, a simple linear model was constructed with this non-sensory score as the sole predictor, and the residuals from the model were treated as "clean" thesholds and used in all analyses thereafter.

Supplementary amplitude modulation (AM) detection task. To further investigate the influence of "non-sensory factors" on behavioral measures in general, we carried out a supplementary experiment using a task that is unrelated to TFS processing - an AM detection task similar to the one used in Bharadwaj et al. (2015). A similar 2AFC procedure as in the FM and ITD detection threshold measurements was employed. The target was a $500-\mathrm{Hz}, 75 \mathrm{~dB}$ SPL band of noise centered at 4 or $8 \mathrm{kHz}$, and amplitude modulated at $19 \mathrm{~Hz}$. Two unmodulated tones, flanked at two equivalent rectangular bandwidths (ERBs) (Glasberg and Moore, 1990; Moore, 1968) away from the center frequency, each at $75 \mathrm{~dB}$ SPL, were used to minimize off-frequency listening. The signal in the reference interval was statistically identical but unmodulated. Using a noise carrier helps eliminate spectral cues for the AM detection task (Viemeister, 1979). The threshold for the modulation depth detection was determined by an adaptive weighted one-up-one-down procedure (Kaernbach, 1991).

\subsubsection{Electrophysiological Measures of the TFS Coding.}

While behavioral measures directly assess perceptual sensitivity to TFS, they may also reflect common "non-sensory" factors such as attention and motivation. To dissociate TFS coding from "non-sensory" factors, we designed two passive EEG measures of the TFS coding and compared them to individual behavioral measures. For EEG measurements, participants watched a silent, captioned video of their choice while passively listening to the auditory stimuli. EEG recordings were obtained using a 32-channel EEG system (Biosemi Active Two), while the stimuli were presented via ER-2 insert earphones.

General EEG setup and pre-processing procedures. The Biosemi EEG system employs active common-mode noise rejection using a pair of ground electrodes in a "drive-right leg" configuration (Metting van Rijn et al., 1990). EEG recordings were re-referenced to the average voltage across the 
two ear lobes. For cortical response analyses (EEG-ITD, see blow), the raw data were bandpass filtered from 1-50 Hz, whereas for subcortical responses (EEG-FFR, see blow), raw data were filtered from 400$1300 \mathrm{~Hz}$. The 400-1300 Hz bandpass filter eliminates artifacts from eye blinks. For the 1-50 Hz cortical data, ocular artifacts were removed using the signal-space projection technique (Uusitalo and Ilmoniemi, 1997). After the eye-blink correction, epochs with large voltage excursions (above $150 \mu \mathrm{V}$ for cortical recordings; above $50 \mu \mathrm{V}$ for subcortical recordings) were excluded to reduce movement artifacts. For both cortical and subcortical recordings, analyses focused on recordings from vertex electrodes (i.e., Fz and $\mathrm{Cz}$ channels).

Cortical correlates of TFS-based ITD processing. Cortical EEG was recorded in response to 70 dB-SPL 500-Hz tones that were amplitude-modulated (100\% depth) at $40.8 \mathrm{~Hz} .40 .8 \mathrm{~Hz}$ can elicit a strong auditory steady-state (ASSR) response in EEG recordings (Picton et al., 2003); this response was used here as a measure of recording quality (Figure 1-C). The stimulus duration was of 1.5 seconds. As with the behavioral measurement, the leading ear for the ITD switched 1-second into the trial. The direction of the ITD switch was randomized across trials. To minimize monaural cues, the ITD switch coincided with a trough of the $40.8 \mathrm{~Hz}$ modulation (Figure 1-A). The magnitude of the ITD jump was randomly chosen to be 20,60, 180, or $540 \mu \mathrm{s}$. This approach mirrors the method used in Papesh et al. (2017), where the stimulus switches between in-phase and out-of-phase states (phase shift of $180^{\circ}$ ). Our measurements involved ITD jumps of 20,60, 180, or $540 \mu s$ in magnitude. The magnitude and direction of the ITD jump were randomized across trials. A total of 1200 trials were presented to the listener. The interstimulus interval was uniformly distributed between 500 and 600 ms. Besides amplitude and latency of the averaged evoked response across trials in each condition, we calculated the inter-trial coherence (ITC), which quantifies the consistency in the phase of the evoked response components across trials. ITC of 0 indicates no phase locking (the response is dominated by background noise), and ITC of 1 indicates perfect phase-consistency across trials (no background noise added to the phase-locked response). Thus, the ITC is directly related to the signal-to-noise ratio of the evoked response (Bharadwaj and Shinn-Cunningham, 2014). The frequency band for ITC analysis was restricted to approximately 1-20 Hz, because it is known that cortical transient-evoked responses primarily consist of low-frequency components, and because we sought to separate these responses from the 40.8-Hz ASSR response.

Frequency Following Response (FFR). Subcortical FFRs were measured in response to tones in a forward-masking stimulus configuration (Verschooten and Joris, 2014). The stimuli consisted of three 
A

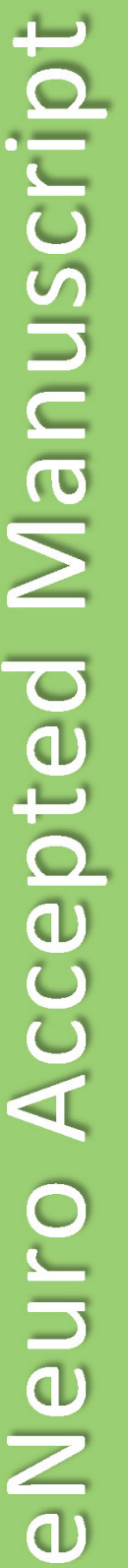

C cor tor gai be 8
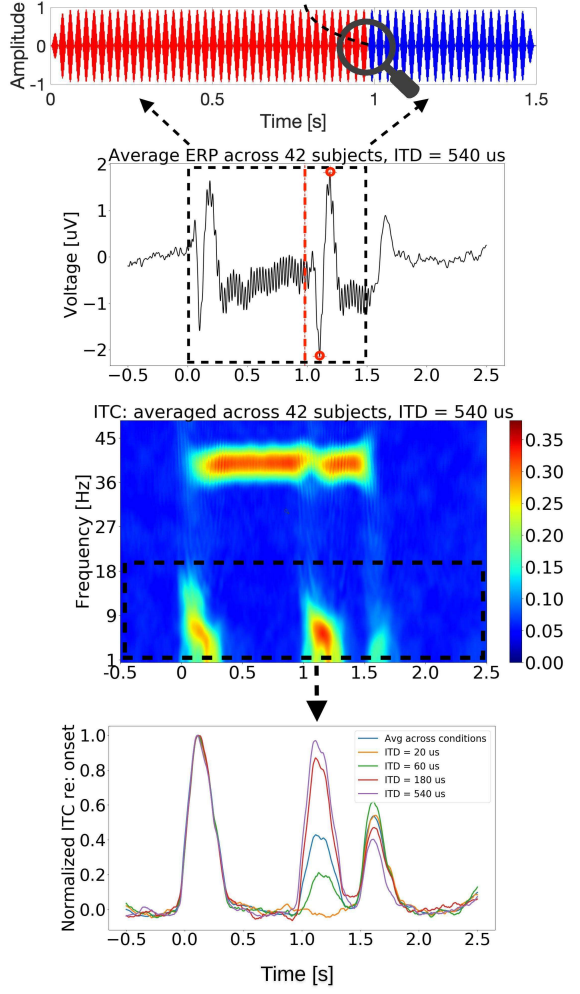

Figure 1. Stimulus paradigm and response from the EEG-TFS sensitivity measurement. A. The stimulus is a 1.5 -second long, $500-\mathrm{Hz}$ pure tone that is amplitude modulated at 40.8 $\mathrm{Hz}$. The red color represents the sound in the right ear, whereas the blue stands for the sound in the left ear. In the figure, the stimulus in the right ear leads in time till 0.98 seconds (indicated by the red segment of zoomed-out view of the stimulus), after which the ITD shifts in polarity, i.e., the stimulus in the left ear takes the lead. The ITD jump occurs when the stimulus amplitude is zero to minimize the involvement of monaural cues (pointed out by the dashed arrow). B. Averaged evoked response potential (ERP) from all trials across 42 subjects in "ITD $=540$ $\mu s$ " condition from $\mathrm{Cz}$ electrode. The red dashed line indicates where the ITD switched polarity, which resulted in N1 and P2 responses (denoted in red dots). C.

Spectrogram of the inter-trial coherence (ITC) of the EEG response, averaged across 42 subjects, with the colormap indicating the ITC. Robust auditory steady-state responses can be seen around the AM frequency of 40.8 $\mathrm{Hz}$. There are also salient responses time locked to the stimulus onset, offset, and importantly, to the ITD jump. D. The average time course of the ITC for frequencies below $20 \mathrm{~Hz}$ is shown for each ITD jump condition. The response evoked by the shift in the ITD polarity increases monotonically with the size of the ITD jump confirming that the response is parametrically modulated by TFS-based processing.

consecutive segments: a 500-Hz probe tone that was 100-ms long and at 75 dB SPL, a "forward-masker" tone of the same frequency and duration but at $85 \mathrm{~dB}$ SPL, and the same probe tone. A 50-ms silent gap was included between the first probe tone and forward-masker, but only a 1-ms gap was included between the forward-masker and the second probe tone. Each stimulus segment was ramped on and off over $5 \mathrm{~ms}$ to reduce audible transitions. The polarity of the stimulus was alternated across a total of 8000 trials. The $500 \mathrm{~Hz}$ component of differential response obtained across the two stimulus polarities 
reflects response components that are phase-locked to the TFS, whereas the summed $500 \mathrm{~Hz}$ response represents the response to the ENV. However, the TFS component can contain both pre-neural (e.g., cochlear microphonic) as well as neural responses. Verschooten and Joris 2014 argued that the nonlinear residual obtained by subtracting the TFS response to the second probe tone from the TFS response to the first probe tone will isolate the neural component and suppress the approximately linear cochlear microphonic $(\mathrm{CM})$. This is because the forward masking of response to the second probe tone only masks the neural component, whereas the CM is intact. Owing to the inner-hair-cell rectification, the summed response across the two polarities also contains a component at twice the stimulus frequency $(1000 \mathrm{~Hz})$ that reflects physiological currents phase-locked to the TFS in the stimulus. Although TFS-related, whether this double-frequency response is purely neural as has been previously interpreted (Parthasarathy et al., 2020), or whether it includes pre-neural contributions is unknown. Thus, we considered two candidate subcortical correlates of TFS processing: (1) The $500 \mathrm{~Hz}$ component derived from the differential response across the two polarities of stimulus presentation, and (2) the $1000 \mathrm{~Hz}$ component derived from the summed response across two polarities of stimulus presentation.

\subsubsection{Statistical Analyses.}

Pearson correlations were calculated to illustrate simple associations between pairs of measurements. Statistical inference about behavior-physiology correlations was made using a multiple linear stepwise regression analysis by adding new potential predictors one by one to model the dependent variable. All reported significant associations met a false discovery rate criterion of $5 \%$ to control for multiple comparisons (Benjamini and Hochberg, 1995). Statistical analyses were performed using R (R Core Team, https://www.r-project.org/).

Code Accessibility. Stimulus generation and data analyses were done using custom scripts. They can be accessed at: https://github.com/AgudemuBorjigin/stimulus-TFS

https://github.com/AgudemuBorjigin/EEGAnalysis, and https://github.com/AgudemuBorjigin/BehaviorDataAnalysis. 


\section{Results}

\subsection{Non-sensory factors contribute to large individual differences in behavioral measures of TFS coding}

Similar to previous reports of large individual differences in the AM and ENV-based ITD detection thresholds across normal-hearing (NH) listeners (Bharadwaj et al., 2015), both the FM and TFS-based ITD detection thresholds varied widely across our NH listeners. FM detection thresholds across $43 \mathrm{NH}$ listeners ranged from 7 to $22 \mathrm{~dB}$ relative to $500 \mathrm{~Hz}$ (i.e., a frequency deviation (Fdev) of 2-13 $\mathrm{Hz}$ from $500 \mathrm{~Hz}$ ). ITD detection thresholds varied from 21 to $39 \mathrm{~dB}$ relative to 1 us (i.e., 11-89 us) across $37 \mathrm{NH}$ listeners. These FM and ITD detection thresholds are shown along with the results from similar studies, in Figure 8 and Figure 9, respectively, and were largely comparable.

Across listeners, neither FM (averaged across two ears) nor ITD thresholds (each averaged across repetitions) correlated with the audiograms (across-ear average of thresholds at $500 \mathrm{~Hz}$; across-ear average of the mean thresholds at high frequencies: 4 and $8 \mathrm{kHz}$ ); however, the two measures were significantly correlated with each other in a simple linear regression analysis $(\mathrm{r}=0.44, \mathrm{p}=0.01, \mathrm{n}=33)$. While the correlations may arise from individual differences in TFS coding, they can also reflect "non-sensory" factors such as attention, motivation, etc. To disambiguate these competing explanations, we assigned each listener a "non-sensory score". When those scores were factored out from each measurement, the correlation between the monaural FM and binaural ITD thresholds dropped such that the association no longer met conventional statistical significance criteria $(R=0.31, P=0.08, n=33)$, suggesting that "nonsensory" factors play a large role in raw scores. Furthermore, when just the blocks with the largest (i.e., worst) FM and ITD thresholds for each subject were compared, considerably stronger correlations were observed ( $\mathrm{r}=0.6, \mathrm{p}=9 \mathrm{e}-4, \mathrm{n}=33)$, underscoring the involvement of "non-sensory" factors in behavioral measurements. Figure 2 shows the correlations between the measured and predicted thresholds solely based on the lapse rates (i.e., the "non-sensory" score). The involvement of "non-sensory" factors is evident, especially for the poorer performers.

To confirm the involvement of non-sensory factors in raw behavioral scores, a similar comparison of thresholds and lapse rates was conducted for the supplementary AM detection task. The predicted thresholds based on the "non-sensory score" significantly correlated with the measured AM thresholds 

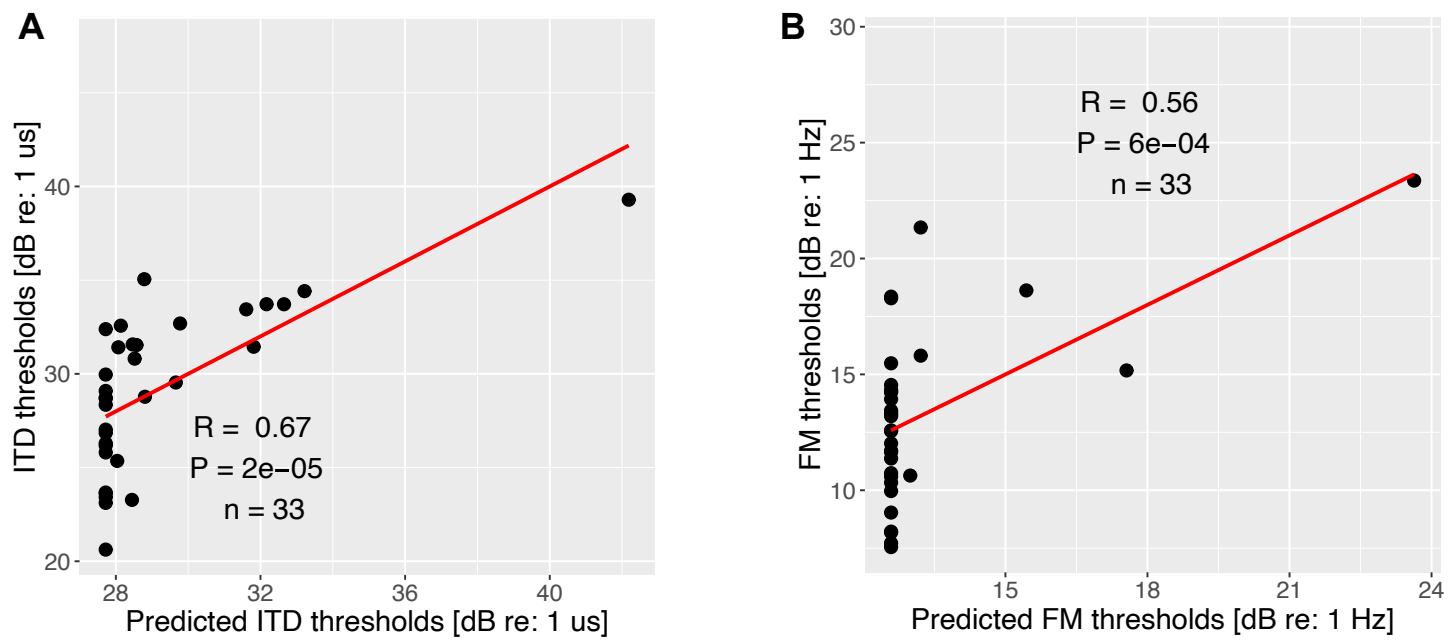

Figure 2. Measured vs. predicted thresholds based on lapse rate. [A] Measured vs predicted ITD detection thresholds; [B] Measured vs predicted FM detection thresholds. The significant contribution of non-sensory factors is apparent, especially for the poorer performers.

$(\mathrm{R}=0.52, \mathrm{P}=1 \mathrm{e}-8, \mathrm{n}=109$; Figure 3$)$. This result indicates the significant weight of "non-sensory"

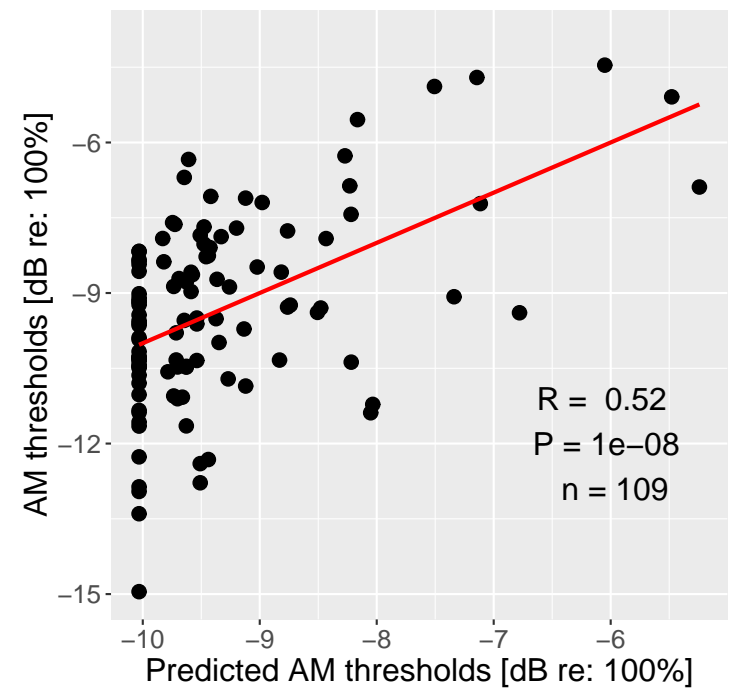

Figure 3. Measured vs predicted AM thresholds based on lapse rate. The thresholds are the average detection thresholds of AM tones at $4 \mathrm{kHz}$ and $8 \mathrm{kHz}$. The significant contribution of non-sensory factors is apparent. 


\subsection{Raw electrophysiological TFS measures are strongly influenced by extraneous} sources of variance

Two passive electrophysiological measurements were carried out to objectively evaluate individual TFS coding. Because passive electrophysiological measures are likely to be influenced by distinct extraneous factors (e.g., head size) compared to behavioral measures (e.g., motivation/engagement), these measurements provide a complementary window into individual TFS coding.

\subsubsection{Candidate cortical correlates of TFS processing}

Cortical responses evoked by the polarity shift of the ITD are quantified through the phase-locking strength shown in the phase-locking spectrograms (Figure $1 \mathrm{C}$ ). Clear responses to the onset, offset, and ITD jump are apparent in the low-frequency portion of the phase-locking spectrogram. The sustained auditory steady-state response (ASSR) is also clear around $40.8 \mathrm{~Hz}$. The average response from $42 \mathrm{NH}$ listeners shows monotonically increasing phase-locking strength of the ITD-evoked response across the ITD magnitudes (Figure 1 D), confirming that the response is indeed sensitive to TFS processing and the size of the ITD jump. Perhaps more important for the search of candidate TFS processing assays, large individual differences are apparent in the phase-locking strength across subjects (Figure 4). Most subjects did not show a salient response for the $20 \mu \mathrm{s}$ condition, and only about half showed robust responses for the $60 \mu \mathrm{s}$ condition. Focusing therefore on the $180 \mu \mathrm{s}$ and $540 \mu \mathrm{s}$ conditions, the $180 \mu \mathrm{s}$ condition is still part of the increasing slope of the response-vs-ITD-jump-size trend, but the response amplitude may have saturated for the $540 \mu s$. Accordingly, we used each individual's response for the $180 \mu s$ condition for comparison to behavior. Note that the ITD being referred to here is the size of the jump; for instance, for the $20 \mu s$ condition, the stimulus started with an ITD of $10 \mu s$ with one ear leading and jumped to the other side about halfway through the stimulus to end with a 10- $\mu s$ ITD with the other ear leading.

Unfortunately, one striking aspect of the result in Figure 4 is that even at $540 \mu s$, the individual differences that were present in the lower ITD conditions persist. The ITD jump is obviously perceptible at $540 \mu \mathrm{s}$, and the EEG response appears to be near saturation level for most individuals; this suggests that a significant portion of the individual differences in the magnitude of the cortical response arises from factors extraneous to TFS-based processing. Extraneous factors that may contribute include anatomical factors such as head size, and the geometry/orientation of the neural sources relative to the scalp sen- 

phase-locked to the TFS.

sors (Bharadwaj et al., 2019). Thus, although the cortical response to ITD jumps is indeed elicited and parametrically modulated by TFS-based processing, raw response amplitude metrics may be unsuitable for use as an individualized assay of TFS coding.

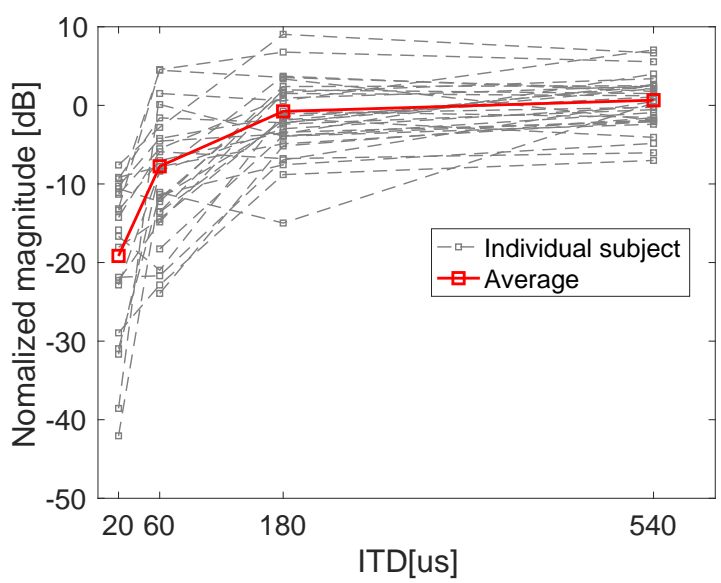

Figure 4. Individual EEG inter-trial coherence (ITC) (averaged under $20 \mathrm{~Hz}$ ) values as a function of the jump size of the ITD. The ITC increases with the ITD for almost all subjects. Robust responses above noise floor are detected for most subjects for the "ITD $=180 \mu \mathrm{s}$ " condition. Interestingly, individual differences present at $180 \mu \mathrm{s}$ persist even at $540 \mu s$ despite the ITD jump being obviously perceptible and the response amplitude appearing to saturate.

\subsection{Candidate subcortical correlates of TFS processing}

Figure 5 shows an example FFR recording from a single individual in response to the stimulus sequence with a probe tone, a forward masker, and a second probe tone. The top row (green traces) shows the differential response across two stimulus polarities. This response to the probe tone (labeled "d1" in Figure 5) tracks the 500-Hz TFS in the stimulus, but contains both pre-neural (e.g., cochlear microphonic) and neural components. Because forward masking is thought to arise from synaptic processing (Verschooten and Joris, 2014), the forward masker would be expected to only suppress the neural (i.e., postsynaptic) component of the response to the second probe tone leaving the pre-neural component intact (labeled "d2" in Figure 5). Thus, subtracting d2 from d1 should leave a purely neural response

The bottom row in Figure 5 (blue traces) shows the summed response across two polarities. Because of inner hair-cell rectification, this response contains a 1000-Hz component arising from the stimulus TFS (also see "Materials and Methods" section). This 1000-Hz component in response to the probe (labeled "s1" in Figure 5) has previously been interpreted as a neural response (Parthasarathy et al., 2020). If that were indeed the case, the forward-masker would considerably suppress the $1000 \mathrm{~Hz}$ component in 
response to the second probe (labeled "s2" in Figure 5).

Figure 6 shows the average d1 (panel A), d1-d2 (panel B), s1 (panel C), and s1-s2 (panel D) response obtained across subjects, quantified in the frequency domain. It is evident from the reduced size of the (d1$\mathrm{d} 2$ ) response compared to the $\mathrm{d} 1$ response, and the reduced size of the (s1 - s2) response compared to the s1 response that, forward masker only has a partially suppressing effect. This provides evidence that both candidate TFS measures - the $500 \mathrm{~Hz}$ component from the difference across stimulus polarities, and the $1000 \mathrm{~Hz}$ component from the sum across stimulus polarities - have significant pre-neural contributions. This is in contrast to the previous interpretation that the component at double the tone frequency is purely neural (Parthasarathy et al., 2020).

These results indicate that a forward-masking paradigm will need to be employed to extract the purely neural "residual" response. Unfortunately, unlike transtympanic recordings that are difficult to perform (Verschooten et al., 2015), this residual is small and not readily measurable from all individual subjects. Thus, while subcortical envelope-following responses (EFRs) provide a robustly measurable correlate of envelope processing (Bharadwaj et al., 2015), tracking the TFS via FFRs are not promising, and not readily measured across all individuals despite our cohort being comprised of NH listeners.

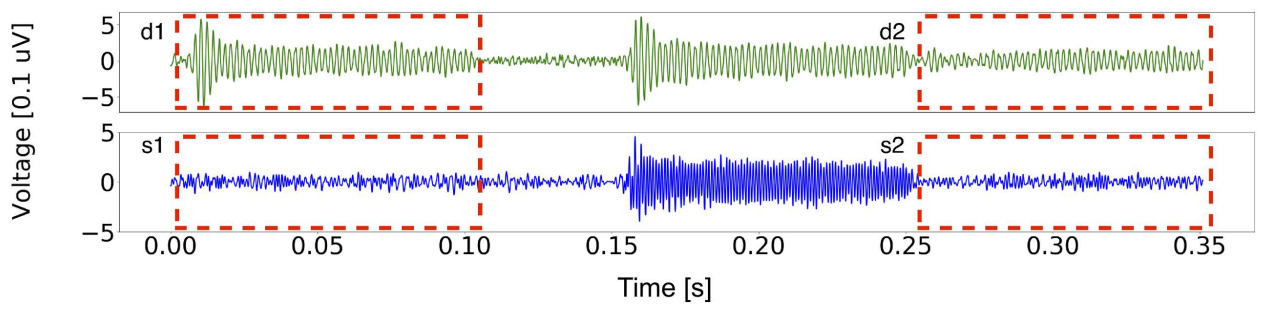

Figure 5. Frequency following response (FFR) to the probe-forward-masker-probe stimulus sequence for an individual subject. The top row (green trace) represents the differential response across two stimulus polarities, whereas the bottom row (blue trace) represents the summed response across two stimulus polarities. The first boxed segments in both rows (red, dashed box, labeled d1 or s1) reflect the raw response to the probe tone, which is likely a mixture of neural and pre-neural responses (e.g., cochlear microphonic; CM), whereas the second boxed segments in both rows (red, dashed box, labeled $\mathrm{d} 2$ or s2) is the adapted response after forward masking. For d2 and s2, the pre-neural (e.g., CM) component is expected to be intact whereas the neural response is attenuated by forward masking (due to a very short 1-ms gap). The forward masker only partially suppresses the responses, suggesting a strong pre-neural contribution to d1 and s1. The weaker residuals obtained by subtraction, i.e., (d1 $\mathrm{d} 2)$ and (s1 - s2) is likely purely neural. 


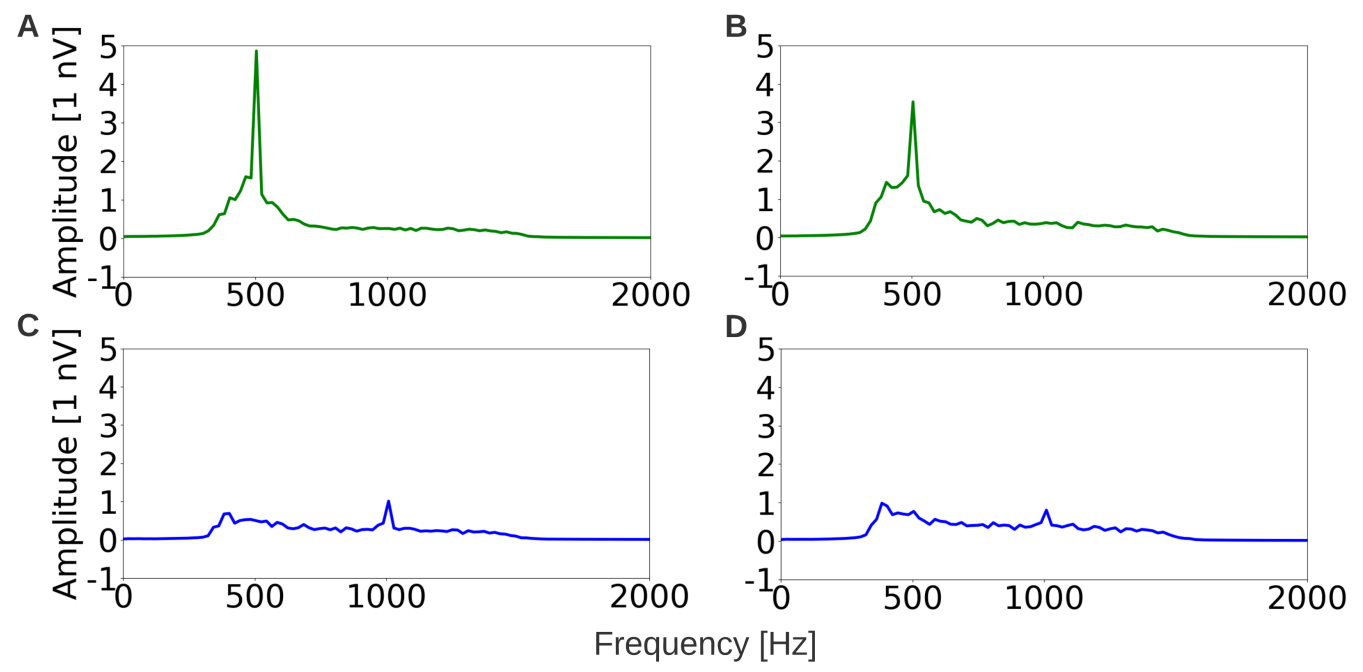

Figure 6. Frequency-domain representations of the d1 (A), d1-d2 (B), s1 (C), and s1-s2 (D) segments from Figure 5, but averaged across subjects. Forward masking partially attenuates both the $500-\mathrm{Hz}$ component of d1 response, and the 1000-Hz component of the s1 response, suggesting that both responses reflect a mix of pre-neural and neural sources.

\section{4 "Adjusted" behavioral and cortical measures are strongly correlated, likely reflecting TFS coding}

Based on individual differences in the cortical amplitude measure persisting for the large-ITD-jump $(540 \mu s)$ condition, we concluded that the amplitude measure of cortical phase-locking was dominated by extraneous variance, likely from anatomic factors. Thus, we focused our attention on the latency of the ITD-jump response, because the latency is expected to be unaffected by the scaling effects of individual anatomy. In particular, we extracted the latency of the cortical response to the $180 \mu s$ jump condition to avoid floor and ceiling effects. The latency was the mean of N1 and P2 latency (the latency is the time difference between the red dashed line and either N1 or P2 peak in Figure 1-B). The use of the latency metric was also motivated by the previous successful use of this EEG-latency measure to predict individual behavioral measures of spatial release from masking (Papesh et al., 2017). In addition to this latency metric, the slope of the cortical-response amplitude with increasing ITD-jump (i.e., the increase from the $60 \mu \mathrm{s}$ condition to the $180 \mu \mathrm{s}$ condition, divided by the $540 \mu \mathrm{s}$ condition, in the ITC plot of Figure 4) was extracted as a normalized measure of TFS processing that would mitigate the overall scaling influence of 
anatomical factors. This normalization was also motivated by the previous successful use of a similarly normalized electrophysiological measure in the context of modulation processing (Bharadwaj et al., 2015).

Both of these "adjusted" cortical measures exhibited significant correlations with behaviorally measured ITD thresholds. Specifically, individual differences in latency of the cortical ITD-jump response (for $180 \mu s)$ correlated with individual differences in the ITD detection thresholds $(\mathrm{R}=0.35, \mathrm{P}=0.048$, $\mathrm{n}=32$ ). The correlation improved when the behavioral scores were also adjusted to factor out the "nonsensory score" $(\mathrm{R}=0.45, \mathrm{P}=0.01, \mathrm{n}=32)$. The slope metric from the cortical EEG response also correlated with ITD thresholds both with and without adjustments to the behavioral scores $(\mathrm{R}=0.43$, $\mathrm{P}=0.021, \mathrm{n}=32$, original ITD scores; $\mathrm{R}=0.42, \mathrm{P}=0.028$, with "non-sensory" score factored out). There were no significant brain-behavior correlations with "unadjusted" or "raw" metrics, such as the ITC amplitude of the ITD-evoked response, even after normalization by the ITC amplitude of the onset response.

With the subcortical measures, because results indicated a significant pre-neural contribution for both candidate TFS measures, and the residual neural component extracted from the forward-masking paradigm was not robustly measurable for many participants, we did not explore FFR-behavior associations in detail. A simple correlational analysis between the residual (d1 - d2) $500 \mathrm{~Hz}$ response and ITD thresholds suggested that the correlations were not statistically distinguishable from zero (not shown).

A multiple linear regression model was used to predict ITD detection thresholds using both the "nonsensory" score, the EEG latency, as well as the EEG normalized slope metric (both from cortical ITDjump response). The model could predict the behavioral ITD threshold well (Figure 7) with the predictors together accounting for more than half of the variance observed in the behavioral thresholds (Table 1). We interpreted this result as suggesting that both "adjusted" behavioral scores, and electrophysiological latency or slope metrics in response to TFS-based binaural processing are promising candidate assays of TFS processing that may be suitable for use at the individual level.

\section{Discussion}

In the present study, we sought to identify viable assays that can index the fidelity of TFS processing at the individual subject level. To obtain insight into whether individual differences in various candidate measures reflected TFS-based processing or extraneous factors, we compared individual differences in 

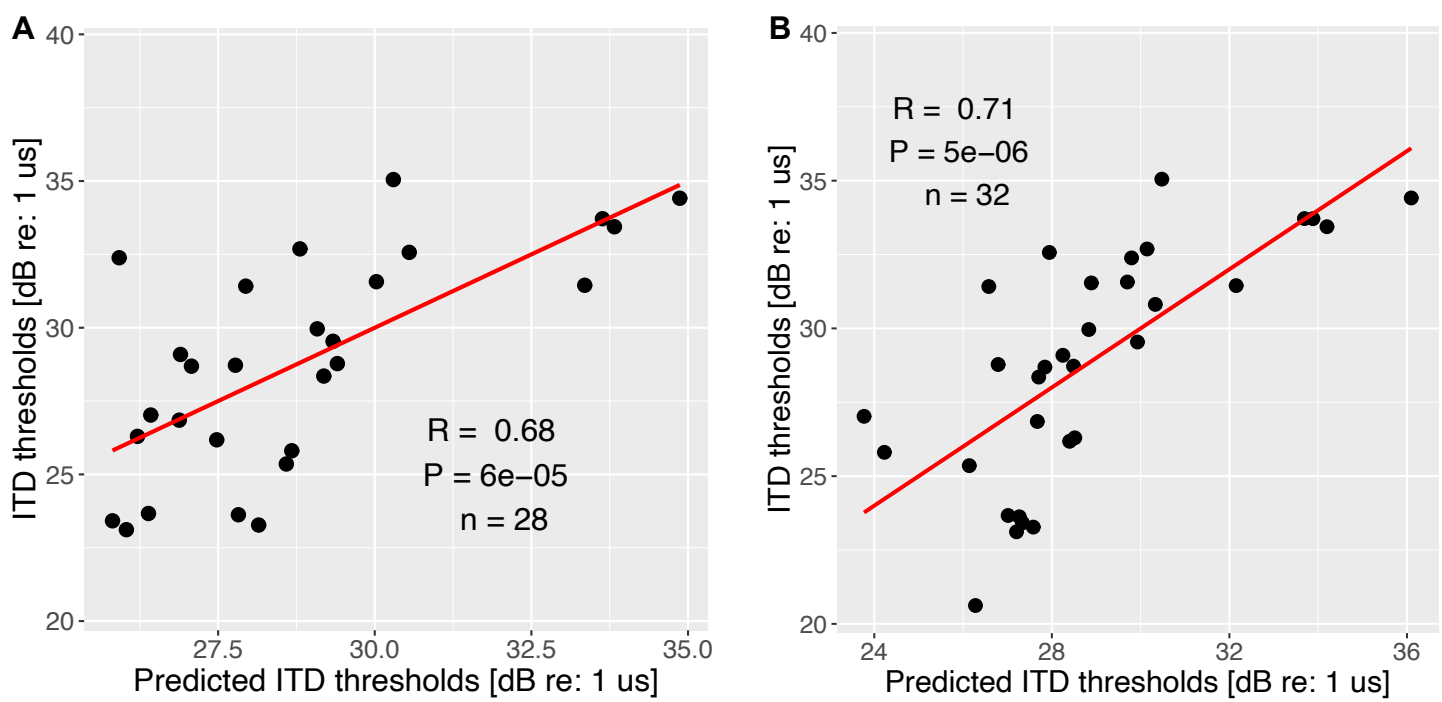

Figure 7. Model prediction of the ITD detection thresholds, based on the combination of lapse rate and slope (60- $\mu s$ to $180-\mu s$ condition) [A], or the combination of lapse rate and EEG latency [B]. Please refer to Table 1 for the variance explained by each factor.

\begin{tabular}{ll}
\hline Predictor & Variance Explained \\
\hline Non-sensory score & $37.48 \%$ \\
EEG latency & $10.03 \%$ \\
EEG slope & $9.28 \%$ \\
\hline Explained & $56.79 \%$ \\
Unexplained & $43.21 \%$ \\
\hline
\end{tabular}

Table 1. Model prediction of the behavioral ITD detection thresholds, with factors including the "non-sensory" score, EEG latency, and EEG slope. The variations accounted for by the "non-sensory" score are more than three times as by either one of the two EEG metrics. Together, more than half the variance can be explained.

behavioral scores across FM and ITD detection tasks to differences in cortical and subcortical EEG-based measures. Results revealed the strong influence of extraneous factors on both behavioral scores and amplitude-based EEG metrics.

With behavioral measures, "non-sensory" factors, quantified using the lapse rate in catch trials, could account for a third of the variance across individuals. Although previous work has explored a range of behavioral TFS measures (Moore and Sek, 2009; Hopkins and Moore, 2010; Sek and Moore, 2012), the results from the present study underscore the importance of adjusting raw behavioral scores to reduce 
the impact of "non-sensory" factors. Indeed, although raw FM and ITD measures correlated significantly with each other, similar to the correlation between monaural AM detection and binaural envelope-ITD thresholds (Bharadwaj et al., 2015), this was driven in part by "non-sensory" factors. Because phaselocking to the TFS is essential for low-frequency ITD processing (Yin and Chan, 1990), it is plausible that ITD detection thresholds can provide an index of TFS sensitivity. On the other hand, whether FM detection relies on TFS coding has been controversial due to the possible role of recovered ENV cues that result from cochlear filtering of FM stimuli; indeed, FM stimuli lead to perceptible out-of-phase ENV fluctuations at cochlear places tuned to frequencies just above and below the FM carrier (Whiteford et al., 2017; Whiteford and Oxenham, 2015). Whiteford et al. (2020) extensively tested the role of place coding in FM detection and found that place coding by itself can account for the observed variations in FM sensitivity across all carrier frequencies and modulation rates. This finding is in contrast to the widely accepted view of the utilization of time coding in the detection of slow-rate FM (Strelcyk and Dau, 2009; Moore and Sek, 1996; Parthasarathy et al., 2020). Together with our finding that non-sensory factors influence raw behavioral scores, this uncertainty about the link between TFS coding and FM detection calls into question the previous use of FM detection scores as a correlate of TFS processing. In contrast, unambiguous theoretical links can be made between ITD detection and TFS coding, suggesting that once ITD thresholds are adjusted to reduce the influence of "non-sensory" scores, they may serve as a useful metric of TFS processing. This was corroborated by our finding that passive EEG measures, when combined with non-sensory scores, can account for more than half of the variance in ITD thresholds. Here, we used lapse rates in the catch trials to obtain a correlate of "non-sensory" factors. Alternately, a surrogate behavioral task that does not rely on TFS coding (e.g., interaural level difference sensitivity) may also be used to adjust ITD thresholds with similar benefits.

Another key finding from the present study is that although passive EEG measurements can potentially reflect TFS-based processing objectively, they too are susceptible to the influence of extraneous factors. Indeed, consistent with the interpretation that individual anatomical factors can have a scaling influence on response amplitudes, we found that cortical responses phase-locked to ITD changes showed large individual differences even for a large ITD jump $(540 \mu s)$ where the response amplitude was near saturation for most individuals. Therefore, we argued that the evoked-response latency and/or percent growth/slope metrics may be better assays of TFS processing. Accordingly, latency and slope metrics showed significant correlations with behavioral ITD detection thresholds. For candidate subcor- 
tical FFR-based measures of TFS processing, our results showed that pre-neural physiological currents (cochlear microphonic, inner hair-cell currents) contribute significantly to the measure, thus complicating their applicability. Indeed, brainstem response measures from individuals with compromised inner hair-cell synaptic transmission show that pre-neural transduction currents can contribute to the measured response (Santarelli et al., 2009). Moreover, when employing a forward-masking-based design to isolate the neural component of the FFR, the resulting signal is relatively weak even in our NH cohort. This result from non-invasive ear-canal recordings is in contrast to neurophonic measurements from the auditory nerve (Snyder and Schreiner, 1985) or round window (Henry, 1995) from animals, or FFR measurements from humans using transtympanic electrodes where the forward-masking design has been used successfully (Verschooten et al., 2018). Although FFRs have previously been used as a putative correlate of TFS-based processing (Parthasarathy et al., 2020), our results suggest that further experiments are needed to clarify the interpretation of those results and to enhance the quality of the measured signal across.

Our finding that the subcortical FFR may be a poor correlate of neural TFS processing is in contrast to previous results suggesting that subcortical envelope-following responses (EFRs) are correlated with behavioral measures of ENV processing. For example, Bharadwaj et al. (2015) showed that the AM detection thresholds and ENV-based ITD thresholds correlated strongly with normalized EFR-based metrics. This is likely both because EFR measurements more readily exclude pre-neural contributions (which primarily track the TFS), and because Bharadwaj et al. (2015) obtained asymptotic behavioral scores from a large number of trials (1200-1500 trials) from trained subjects. Indeed, with naïve subjects in this study, an AM detection task similar to the one used in Bharadwaj et al. (2015) also showed a strong influence of "non-sensory" factors.

In summary, the present study examined various candidate assays for quantifying TFS processing at the individual subject level. These included behavioral FM and ITD detection thresholds, EEG-based cortical and subcortical physiological measures. Among these, our experiments suggest that the latency of cortical responses to ITD jumps, normalized cortical response amplitude (i.e., percent growth/slope), and "adjusted" ITD thresholds may all be useful. Indeed, when a multiple linear regression model was constructed to predict behavioral ITD thresholds, the combination of the "non-sensory" score (lapse rate in catch trials), EEG latency, and slope measures could account for more than $50 \%$ of the variance across individuals. Our results are consistent with the findings by Papesh et al. (2017), who also found a 
correlation between ITD-evoked EEG latency and spatial-hearing outcomes such as spatial release from masking. Given that multiple candidate measures were explored to identify the most promising assays, future experiments should be conducted to independently confirm the efficacy of the assays endorsed by our results. The most promising assays rely on binaural TFS-based processing. Indeed, similarly to our results, steady-state cortical responses that track continuous interaural phase modulations have also been found to correlate with behavioral binaural sensitivity, further corroborating the potential utility of cortical binaural measures as electrophysiological assays of TFS processing (Koerner et al., 2020; Undurraga et al., 2016)

Reliable measures of TFS processing are critical for future investigations into the role of the TFS in everyday hearing using intact speech-in-noise stimuli without vocoding manipulations. While sub-band vocoding can allow for independent manipulation of acoustic TFS and envelope cues, subsequent cochlear processing can confound these factors once again (Gilbert and Lorenzi, 2006; Swaminathan and Heinz, 2012). Furthermore, when both rate-place/ENV cues and TFS cues are redundant information, vocoding experiments cannot provide insight into how they are perceptually weighted. The candidate TFS measures identified in the present study can help address these gaps.

\section{References}

Ardoint, M. and Lorenzi, C. (2010). Effects of lowpass and highpass filtering on the intelligibility of speech based on temporal fine structure or envelope cues. Hearing Research, 260(1):89-95.

Benjamini, Y. and Hochberg, Y. (1995). Controlling the False Discovery Rate: A Practical and Powerful Approach to Multiple Testing. Royal Statistical Society.

Bernstein, J. G. and Oxenham, A. J. (2003). Pitch discrimination of diotic and dichotic tone complexes: Harmonic resolvability or harmonic number? J Acoust Soc Am, 113(6):3323-3334.

Bernstein, L. R. and Trahiotis, C. (2002). Enhancing sensitivity to interaural delays at high frequencies by using "transposed stimuli". The Journal of the Acoustical Society of America, 112(3):1026-1036.

Best, V., Ozmeral, E., Gallun, F. J., Sen, K., and Shinn-Cunningham, B. G. (2005). Spatial unmasking 
of birdsong in human listeners: Energetic and informational factors. The Journal of the Acoustical Society of America, 118(6):3766-3773.

Bharadwaj, H. M., Mai, A. R., Simpson, J. M., Choi, I., Heinz, M. G., and Shinn-Cunningham, B. G. (2019). Non-invasive assays of cochlear synaptopathy-candidates and considerations. Neuroscience, $407: 53-66$.

Bharadwaj, H. M., Masud, S., Mehraei, G., Verhulst, S., and Shinn-Cunningham, B. G. (2015). Individual Differences Reveal Correlates of Hidden Hearing Deficits. Journal of Neuroscience, 35(5):2161-2172.

Bharadwaj, H. M. and Shinn-Cunningham, B. G. (2014). Rapid acquisition of auditory subcortical steady-state responses using multichannel recordings. Clin Neurophysiol, 125(9):1878-1888.

Brughera, A., Dunai, L., and Hartmann, W. M. (2013). Human interaural time difference thresholds for sine tones: The high-frequency limit. The Journal of the Acoustical Society of America, 133(5):28392855.

Buss, E., Hall, J. W. I., and Grose, J. H. (2004). Temporal Fine-Structure Cues to Speech and Pure Tone Modulation in Observers with Sensorineural Hearing Loss. Ear and Hearing, 25(3):242-250.

Drullman, R. (1995). Temporal envelope and fine structure cues for speech intelligibility. J Acoust Soc $A m, 97(1): 585-592$.

Dye, R. H. (1990). The combination of interaural information across frequencies: Lateralization on the basis of interaural delay. The Journal of the Acoustical Society of America, 88(5):2159-2170.

Gilbert, G. and Lorenzi, C. (2006). The ability of listeners to use recovered envelope cues from speech fine structure. The Journal of the Acoustical Society of America, 119(4):2438-2444.

Glasberg, B. R. and Moore, B. C. J. (1990). Derivation of auditory filter shapes from notched-noise data. Hearing Research, 47(1):103-138.

Grose, J. H. and Mamo, S. K. (2010). Processing of temporal fine structure as a function of age. Ear and hearing, 31(6):755-760.

Grose, J. H. and Mamo, S. K. (2012). Frequency modulation detection as a measure of temporal processing: Age-related monaural and binaural effects. Hearing Research, 294(1):49-54. 
Harris, J. D. (1952). Pitch Discrimination. The Journal of the Acoustical Society of America, 24(6):750755. Publisher: Acoustical Society of America.

Hasenstaub, A., Otte, S., Callaway, E., and Sejnowski, T. J. (2010). Metabolic cost as a unifying principle governing neuronal biophysics. Proceedings of the National Academy of Sciences, 107(27):12329-12334. Publisher: National Academy of Sciences Section: Biological Sciences.

He, N.-j., Dubno, J. R., and Mills, J. H. (1998). Frequency and intensity discrimination measured in a maximum-likelihood procedure from young and aged normal-hearing subjects. The Journal of the Acoustical Society of America, 103(1):553-565. Publisher: Acoustical Society of America.

Henning, G. B. (1983). Lateralization of low-frequency transients. Hearing Research, 9(2):153-172.

Henry, K. R. (1995). Auditory nerve neurophonic recorded from the round window of the Mongolian gerbil. Hearing Research, 90(1):176-184.

Hershkowitz, R. M. and Durlach, N. I. (1969). Interaural Time and Amplitude jnds for a 500-Hz Tone. The Journal of the Acoustical Society of America, 46(6B):1464-1467.

Hilbert, D. (1906). Grundzüge einer allgemeinen Theorie der linearen Integralgleichungen. Vierte Mitteilung. Nachrichten von der Gesellschaft der Wissenschaften zu Göttingen, MathematischPhysikalische Klasse, 1906:157-228.

Hopkins, K. and Moore, B. C. J. (2009). The contribution of temporal fine structure to the intelligibility of speech in steady and modulated noise. The Journal of the Acoustical Society of America, 125(1):442446. Publisher: Acoustical Society of America.

Hopkins, K. and Moore, B. C. J. (2010). Development of a fast method for measuring sensitivity to temporal fine structure information at low frequencies. International Journal of Audiology, 49(12):940946. Publisher: Taylor \& Francis_eprint: https://doi.org/10.3109/14992027.2010.512613.

Hopkins, K., Moore, B. C. J., and Stone, M. A. (2008). Effects of moderate cochlear hearing loss on the ability to benefit from temporal fine structure information in speech. The Journal of the Acoustical Society of America, 123(2):1140-1153. Publisher: Acoustical Society of America. 
Houtsma, A. J. and Smurzynski, J. (1990). Pitch identification and discrimination for complex tones with many harmonics. J Acoust Soc Am, 87(1):304-310.

Ihlefeld, A. and Shinn-Cunningham, B. G. (2011). Effect of source spectrum on sound localization in an everyday reverberant room. The Journal of the Acoustical Society of America, 130(1):324-333.

Johnson, D. H. (1980). The relationship between spike rate and synchrony in responses of auditory-nerve fibers to single tones. J Acoust Soc Am, 68(4):1115-1122.

Joris, P., Schreiner, C., and Rees, A. (2004). Neural processing of amplitude-modulated sounds. Physiol Rev, 84(2):541-578.

Joris, P. X. and Yin, T. C. (1992). Responses to amplitude-modulated tones in the auditory nerve of the cat. J Acoust Soc Am, 91(1):215-232.

Kaernbach, C. (1991). Simple adaptive testing with the weighted up-down method. Atten Percept Psychophys, 49(3):227-229.

Kidd, G., Watson, C., and Gygi, B. (2007). Individual differences in auditory abilities. J Acoust Soc Am, 122(1):418-435.

Klumpp, R. G. and Eady, H. R. (1956). Some Measurements of Interaural Time Difference Thresholds. The Journal of the Acoustical Society of America, 28(5):859-860.

Koerner, T. K., Muralimanohar, R. K., Gallun, F. J., and Billings, C. J. (2020). Age-Related Deficits in Electrophysiological and Behavioral Measures of Binaural Temporal Processing. Frontiers in Neuroscience, 14:1105.

Laughlin, S. B., de Ruyter van Steveninck, R. R., and Anderson, J. C. (1998). The metabolic cost of neural information. Nature Neuroscience, 1(1):36-41. Number: 1 Publisher: Nature Publishing Group.

Lelo de Larrea-Mancera, E. S., Stavropoulos, T., Hoover, E. C., Eddins, D. A., Gallun, F. J., and Seitz, A. R. (2020). Portable Automated Rapid Testing (PART) for auditory assessment: Validation in a young adult normal-hearing population. The Journal of the Acoustical Society of America, 148(4):1831-1851. Publisher: Acoustical Society of America. 
Lorenzi, C., Debruille, L., Garnier, S., Fleuriot, P., and Moore, B. C. J. (2009). Abnormal processing of temporal fine structure in speech for frequencies where absolute thresholds are normal. The Journal of the Acoustical Society of America, 125(1):27-30. Publisher: Acoustical Society of America.

McDermott, J. H., Lehr, A. J., and Oxenham, A. J. (2010). Individual differences reveal the basis of consonance. Current Biology, 20(11):1035-1041.

Metting van Rijn, A., Peper, A., and Grimbergen, C. (1990). High-quality recording of bioelectric events. Med Biol Eng Comput, 28:389-397.

Moore, B. C. (1973). Frequency difference limens for short-duration tones. J Acoust Soc Am, 54(3):610619.

Moore, B. C. J. (1968). Parallels betwen frequency selectivity measured psychophysically and in cochlear mechanics.

Moore, B. C. J. and Sek, A. (1996). Detection of frequency modulation at low modulation rates: Evidence for a mechanism based on phase locking. The Journal of the Acoustical Society of America, 100(4):2320 2331.

Moore, B. C. J. and Sek, A. (2009). Development of a fast method for determining sensitivity to temporal fine structure. International Journal of Audiology, 48(4):161-171. Publisher: Taylor \& Francis _eprint: https://doi.org/10.1080/14992020802475235.

Oxenham, A. J. (2012). Pitch perception. J Neurosci, 32(39):13335-13338.

Oxenham, A. J. (2013). Revisiting place and temporal theories of pitch. Acoustical Science and Technology, $34(6): 388-396$.

Oxenham, A. J. and Simonson, A. M. (2009). Masking release for low-and high-pass-filtered speech in the presence of noise and single-talker interference. J Acoust Soc Am, 125(1):457-468.

Papesh, M. A., Folmer, R. L., and Gallun, F. J. (2017). Cortical Measures of Binaural Processing Predict Spatial Release from Masking Performance. Frontiers in Human Neuroscience, 11. 
Parthasarathy, A., Hancock, K. E., Bennett, K., DeGruttola, V., and Polley, D. B. (2020). Bottom-up and top-down neural signatures of disordered multi-talker speech perception in adults with normal hearing. eLife, 9:e51419. Publisher: eLife Sciences Publications, Ltd.

Picton, T. W., John, M. S., Dimitrijevic, A., and Purcell, D. (2003). Human auditory steady-state responses: Respuestas auditivas de estado estable en humanos. International Journal of Audiology, 42(4):177-219.

Ruggles, D., Bharadwaj, H., and Shinn-Cunningham, B. G. (2011). Normal hearing is not enough to guarantee robust encoding of suprathreshold features important in everyday communication. Proceedings of the National Academy of Sciences, 108(37):15516-15521.

Santarelli, R., Del Castillo, I., Rodríguez-Ballesteros, M., Scimemi, P., Cama, E., Arslan, E., and Starr, A. (2009). Abnormal cochlear potentials from deaf patients with mutations in the otoferlin gene. Journal of the Association for Research in Otolaryngology, 10(4):545-556.

Sek, A. and Moore, B. C. (2012). Implementation of two tests for measuring sensitivity to temporal fine structure. Int J Audiol, 51(1):58-63.

Shower, E. G. and Biddulph, R. (1931). DIFFERENTIAL PITCH SENSITIVITY OF THE EAR. page 14.

Smith, Z. M., Delgutte, B., and Oxenham, A. J. (2002). Chimaeric sounds reveal dichotomies in auditory perception. Nature, 416(6876):87.

Snyder, R. L. and Schreiner, C. E. (1985). Forward masking of the auditory nerve neurophonic (ANN) and the frequency following response (FFR). Hearing Research, 20(1):45-62.

Strelcyk, O. and Dau, T. (2009). Relations between frequency selectivity, temporal fine-structure processing, and speech reception in impaired hearing. The Journal of the Acoustical Society of America, $125(5): 3328-3345$.

Swaminathan, J. and Heinz, M. G. (2012). Psychophysiological Analyses Demonstrate the Importance of Neural Envelope Coding for Speech Perception in Noise. Journal of Neuroscience, 32(5):1747-1756.

Undurraga, J. A., Haywood, N. R., Marquardt, T., and McAlpine, D. (2016). Neural Representation of 
Interaural Time Differences in Humans - an Objective Measure that Matches Behavioural Performance. Journal of the Association for Research in Otolaryngology, 17(6):591-607.

Uusitalo, M. A. and Ilmoniemi, R. J. (1997). Signal-space projection method for separating meg or eeg into components. Medical and biological engineering and computing, 35(2):135-140.

Verschooten, E., Desloovere, C., and Joris, P. X. (2018). High-resolution frequency tuning but not temporal coding in the human cochlea. PLOS Biology, 16(10):e2005164.

Verschooten, E. and Joris, P. X. (2014). Estimation of Neural Phase Locking from Stimulus-Evoked Potentials. Journal of the Association for Research in Otolaryngology, 15(5):767-787.

Verschooten, E., Robles, L., and Joris, P. X. (2015). Assessment of the limits of neural phase-locking using mass potentials. J Neurosci, 35(5):2255-2268.

Verschooten, E., Shamma, S., Oxenham, A. J., Moore, B. C. J., Joris, P. X., Heinz, M. G., and Plack, C. J. (2019). The upper frequency limit for the use of phase locking to code temporal fine structure in humans: A compilation of viewpoints. Hearing Research, 377:109-121.

Viemeister, N. F. (1979). Temporal modulation transfer functions based upon modulation thresholds. The Journal of the Acoustical Society of America, 66(5):1364-1380. Publisher: Acoustical Society of America.

Viswanathan, V., Bharadwaj, H. M., Shinn-Cunningham, B. G., and Heinz, M. G. (2021). Modulation masking and fine structure shape neural envelope coding to predict speech intelligibility across diverse listening conditions. bioRxiv.

Whiteford, K. L., Kreft, H. A., and Oxenham, A. J. (2017). Assessing the Role of Place and Timing Cues in Coding Frequency and Amplitude Modulation as a Function of Age. Journal of the Association for Research in Otolaryngology, 18(4):619-633.

Whiteford, K. L., Kreft, H. A., and Oxenham, A. J. (2020). The role of cochlear place coding in the perception of frequency modulation. eLife, 9:e58468. Publisher: eLife Sciences Publications, Ltd.

Whiteford, K. L. and Oxenham, A. J. (2015). Using individual differences to test the role of temporal 
and place cues in coding frequency modulation. The Journal of the Acoustical Society of America, 138(5):3093-3104.

${ }_{625}$ Yin, T. C. and Chan, J. C. (1990). Interaural time sensitivity in medial superior olive of cat. Journal of Neurophysiology, 64(2):465-488.

${ }_{627}$ Zwicker, E. (1956). Die elementaren Grundlagen zur Bestimmung der Informationskapazität des Gehörs. 


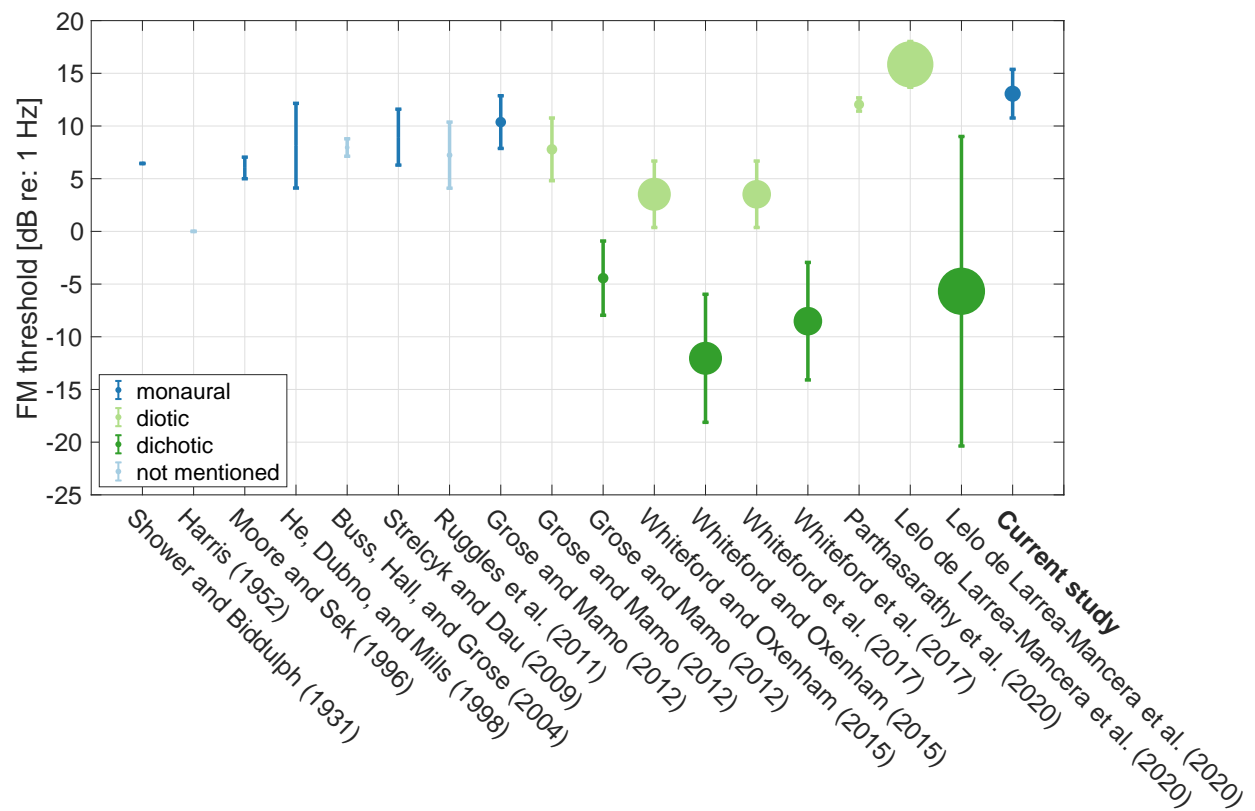

Figure 8. A sample of published reports of FM detection thresholds for comparison (Shower and Biddulph, 1931; Harris, 1952; Moore and Sek, 1996; He et al., 1998; Buss et al., 2004; Strelcyk and Dau, 2009; Ruggles et al., 2011; Grose and Mamo, 2012; Whiteford and Oxenham, 2015; Whiteford et al., 2017; Parthasarathy et al., 2020; Lelo de Larrea-Mancera et al., 2020). Error bar is 1 standard deviation (std). The size of the dot represents the number of subjects (Whiteford and Oxenham (2015) has the most subjects; $\mathrm{N}=100$ ). Stimulus parameters such as stimulus level, carrier frequency, and modulation frequency in the cited studies are similar to those used in the current study, with slight differences (e.g., Ruggles et al. (2011); Strelcyk and Dau (2009) used carrier at $750 \mathrm{~Hz}$ ). Some threshold values are approximate from figures (e.g., mean and std had to be estimated based on median and range in the box whisker plots in Whiteford and Oxenham (2015) and Whiteford et al. (2017)). The mean and std from the young and middle-aged group from Grose and Mamo (2012) were combined to generate a single data point. Some authors expressed the threshold in terms of $\Delta F / F_{c}$, where $\Delta F$ is frequency deviation, and $F_{c}$ is the carrier frequency.

Moore and Sek (1996) used $\Delta F$ that was in two directions, i.e., peak-peak. Subjects from some studies were highly experienced in psychoacoustic tasks hence the thresholds were very low/good. Whiteford and Oxenham (2015); Whiteford et al. (2017) obtained thresholds that fall in the lower end of the results of the current study from a very large number of subjects. This may be because their subjects were younger normal-hearing listeners and the stimuli were presented diotically and dichotically instead of monaurally. 


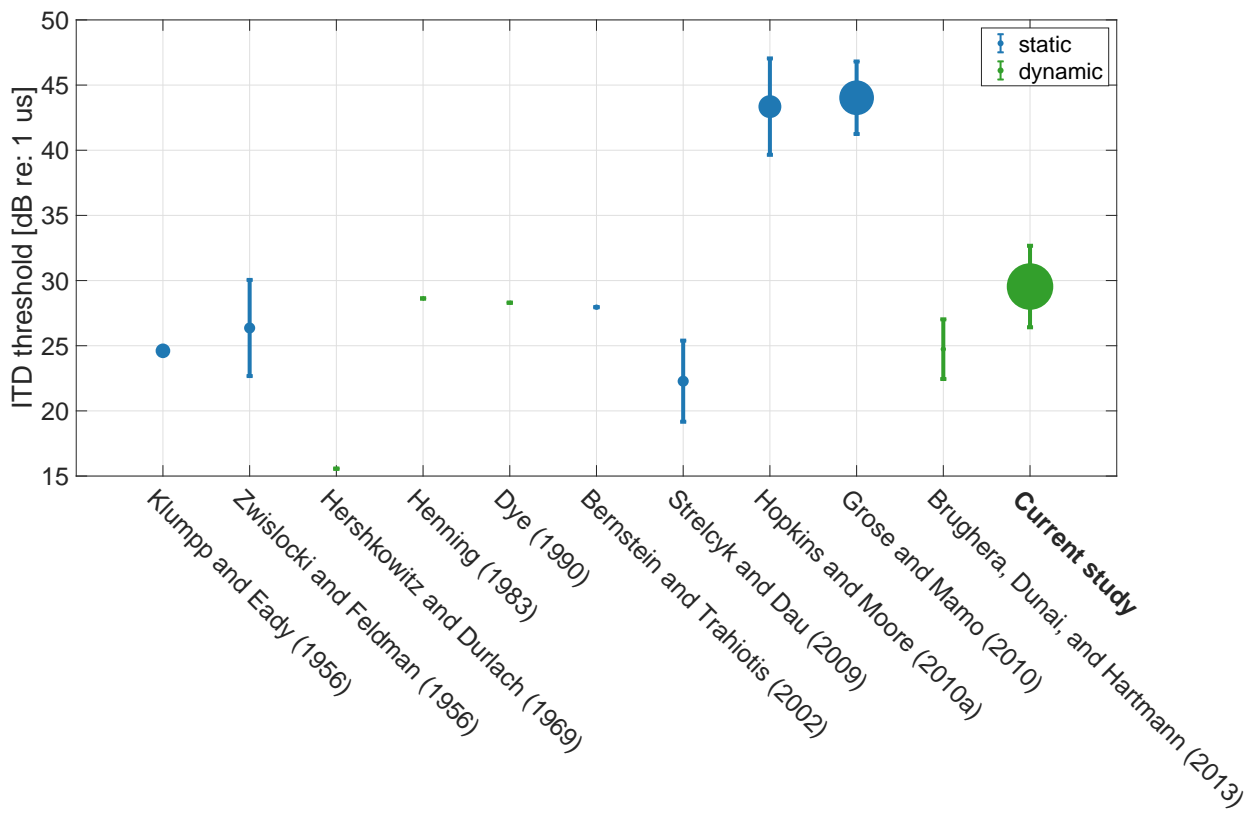

Figure 9. A sample of published reports of ITD detection thresholds for comparison (Klumpp and Eady, 1956; Zwicker, 1956; Hershkowitz and Durlach, 1969; Henning, 1983; Dye, 1990; Bernstein and Trahiotis, 2002; Strelcyk and Dau, 2009; Hopkins and Moore, 2010; Grose and Mamo, 2010; Brughera et al., 2013). Error bar is 1 standard deviation (std). The size of the dot represents the number of subjects (the current study has the most subjects; $N=36$ ). Stimulus parameters such as level and carrier frequency in the cited studies are similar to those used in the current study, with slight differences (e.g., Strelcyk and Dau (2009) used carrier at $750 \mathrm{~Hz}$ ). Note that some threshold values were extracted approximately from figures rather than direct numerical reports. Some of the studies used stimuli with the leading ear switching from one size to the other (labeled "dynamic", marked in green color), whereas others presented an ITD only in the target intervals, with the reference being the midline (labeled "static", marked in blue color). Note that the values from Hershkowitz and Durlach (1969); Brughera et al. (2013) were halved since the authors used ITD/2 in each interval. The mean and std from young and middle-aged cohort from Grose and Mamo (2010) were combined to generate a single data point. Subjects from some studies were highly experienced in psychoacoustic tasks. 